\title{
Asphyxia Aggravates Systemic Hypotension But Not Pulmonary Hypertension in Piglets with Meconium Aspiration
}

\author{
MINNA AALTONEN, HANNA SOUKKA, LAURI HALKOLA, JARMO JALONEN, \\ IRMA E. HOLOPAINEN, PENTTI KERO, AND PEKKA O. KAAPA \\ Research Centre of Applied and Preventive Cardiovascular Medicine [M.A., H.S., L.H., J.J., P.O.K.], \\ Department of Pharmacology and Clinical Pharmacology [I.E.H.], University of Turku, Turku, Finland; \\ Department of Pediatrics, University Hospital of Turku, Turku, Finland [M.A., H.S., P.K.]
}

\section{ABSTRACT}

\begin{abstract}
Meconium aspiration and birth asphyxia are both separately connected to significant pulmonary and systemic hemodynamic changes in newborns, but, although these insults frequently coexist, their combined effects on the neonatal circulation are still controversial. To determine the pulmonary and systemic circulatory changes induced by pulmonary meconium contamination with concurrent asphyxia, 41 anesthetized and ventilated newborn piglets $(10-12 \mathrm{~d})$ were studied for $6 \mathrm{~h}$. Eleven piglets were instilled with a bolus of human meconium intratracheally, and 10 piglets had meconium instillation with immediate induction of an asphyxic insult. Eight piglets had only asphyxia and 12 ventilated piglets served as controls. Meconium instillation (with and without asphyxia) resulted in a sustained decrease in the oxygenation, which remained, however, on the control level in the asphyxic group. Although meconium insufflation (with and without asphyxia) increased pulmonary artery pressure and vascular resistance progressively during the study period, the meconium-induced hypertensive effect was actually diminished by additional asphyxia. Asphyxia alone did not have any effect on these pulmonary hemodynamic parameters. On the other hand,
\end{abstract}

whereas systemic arterial pressure and vascular resistance remained on the control level after meconium instillation alone, asphyxia (with and without pulmonary meconium insult) resulted in a sustained fall in systemic pressure already by $4 \mathrm{~h}$. Our data thus indicate that although the coexisting asphyxia seems to moderate the meconium aspiration-induced pulmonary hypertensive response, this additional asphyxic insult does not affect the associated hypoxemia, but rather significantly exacerbates systemic hypotension. (Pediatr Res 53: 473-478, 2003)
CO, cardiac output
Abbreviations
CVP, central venous pressure
HR, heart rate
MABP, mean arterial blood pressure
MAS, meconium aspiration syndrome
MPAP, mean pulmonary artery pressure
PVR, pulmonary vascular resistance
PWP, pulmonary wedge pressure
SVR, systemic vascular resistance

Aspiration of meconium-stained amniotic fluid frequently produces a severe respiratory failure in term and postterm infants. The clinical MAS has high morbidity and mortality, often linked with the presence of pulmonary hypertension (1-5). The pathophysiology and predisposing factors of the pulmonary hypertensive reaction in MAS are, however, complex and poorly defined. Although abnormal pulmonary arteriolar hypertrophy resulting from intrauterine hypoxia is supposed to play an etiologic role in some fatal cases of

Received November 5, 2001; accepted March 3, 2002.

Correspondence: Minna Aaltonen, M.D., Research Centre of Applied and Preventive Cardiovascular Medicine (CAPC), Kiinamyllynkatu 10, FIN-20520 Turku, Finland; email:minkopo@utu.fi

Supported by the Foundation for Pediatric Research, Finland; Sigrid Juselius Foundation, Finland; and AGA Ab, Sweden.

DOI: 10.1203/01.PDR.0000049514.02607.03 hypertensive MAS (6), experimental data indicate that acute intrapulmonary meconium contamination per se is able to cause, in a concentration-dependent manner, progressive pulmonary hypertensive response $(2,7)$. Alike, there are clinical data suggesting that meconium aspiration and its associated complications, rather than the structural vascular changes, are primarily responsible for the elevated pulmonary vasomotor tone and hypoxemic respiratory failure in newborns $(8,9)$. Because eventual disturbances in cardiac function may additionally cause progressive clinical deterioration of the affected infants, the hemodynamic manifestations in complicated MAS need to be better defined (9).

Similar to severe MAS, birth asphyxia is a further important cause of neonatal morbidity and mortality (10). Severe perinatal asphyxia has been additionally related to neonatal MAS with critical respiratory failure and pulmonary hypertension, 
often refractory to various forms of treatment $(3,11,12)$. Although an acute asphyxic insult at birth may impair the cardiorespiratory function and ultimately contribute to multiorgan involvement $(10,13)$, the clinical, particularly hemodynamic, consequences of asphyxia in infants with an already compromised respiratory system are still controversial $(3,11$, 14-16). We hypothesized that significant asphyxia at birth could exacerbate the cardiorespiratory status in infants with concurrent meconium-induced parenchymal lung disease and thus decided to investigate the early pulmonary and systemic hemodynamic derangements and their interactions in meconium aspiration complicated by acute asphyxia in newborn piglets.

\section{MATERIALS AND METHODS}

Animals. Forty-one 10- to 12-d-old piglets were studied (mean weight $4.0 \mathrm{~kg}$, range $3.6-4.5 \mathrm{~kg}$ ). The animals were premedicated with ketamine hydrochloride $(40 \mathrm{mg} / \mathrm{kg}$ i.m.). They were then intubated with a $5-\mathrm{mm}$ endotracheal tube and ventilated using a pressure-controlled ventilator (Baby-Bird, Palm Springs, CA, U.S.A.). The ventilatory assistance was started with room air at a ventilatory rate of 26 breaths per minute, proximal airway pressure of $24 \mathrm{~cm} \mathrm{H}_{2} \mathrm{O}$, and endexpiratory pressure of $4 \mathrm{~cm} \mathrm{H}_{2} \mathrm{O}$. Anesthesia was maintained with a continuous infusion of pentobarbital $10 \mathrm{mg} / \mathrm{kg} / \mathrm{h}$ i.v. Paralysis was induced with pancuronium bromide $(0.1 \mathrm{mg} / \mathrm{kg})$ and maintained with a continuous infusion $(0.25 \mathrm{mg} / \mathrm{kg} / \mathrm{h}$ i.v. $)$. All the experiments were approved by the Committee of Animal Care in Research of the University of Turku. Animals were cared for in accordance with the procedures outlined in the National Institutes of Health "Guide for the Care and Use of Laboratory Animals."

Physiologic measurements. A 5F Swan-Ganz thermodilution catheter (Baxter Corp., Irvine, CA, U.S.A.) was inserted via the right external jugular vein into the pulmonary artery under intravascular pressure monitoring to measure the MPAP, PWP, and CVP at end expiration. The MABP was monitored and blood samples collected from a small polyethylene catheter, which was placed in the abdominal aorta via the right femoral artery. Pressures were measured using Baxter Uniflow transducers (Model 43-600, Bentley Laboratories, Uden, Holland) and a hemodynamic and respiratory computer system (Patient Data Monitor 565A, Kone, Helsinki, Finland). HR was detected with a transthoracic electrocardiogram. $\mathrm{CO}$ was determined by the thermodilution technique (Model 952A, American Edwards Laboratories, Santa Ana, CA, U.S.A.). The mean value of three measurements, which were within $10 \%$ of each other, was recorded. PVR was calculated from the difference between MPAP and PWP divided by CO and the SVR from the difference between MABP and CVP divided by CO.

The measurements of $\mathrm{pH}$, arterial $\mathrm{CO}_{2}$ pressure $\left(\mathrm{PaCO}_{2}\right)$, $\mathrm{PaO}_{2}$ and oxygen saturation were made by an automatic blood gas analyzer (Radiometer ABL 50, Copenhagen, Denmark) immediately after obtaining the arterial samples. Blood glucose concentration was measured by an amperometric analyzer (Super Glucocard II, GT-1640, Arkray Factory Inc, Shiga, Japan).
Study protocol. After instrumentation, the piglets were stabilized for $1 \mathrm{~h}$ before the baseline measurements. To evaluate the hemodynamic effects of meconium aspiration, 21 piglets were instilled with a bolus $(3 \mathrm{~mL} / \mathrm{kg})$ of human meconium $(65$ $\mathrm{mg}$ meconium $/ \mathrm{mL}$ saline) through the endotracheal tube. The meconium was obtained from the first stools of several healthy term human infants. It was then pooled, lyophilized, and irradiated and, before the experiment, diluted with sterile saline. Seven piglets with a same amount of sterile saline administration served as controls to meconium instillation. To define the hemodynamic effects of asphyxia 18 piglets, including 10 with previous meconium instillation, were studied. The asphyxic insult was originally induced by discontinuing the ventilatory assistance until the MABP fell $>40 \%$ from the baseline level or HR dropped to 70 beats per minute. However, because our preliminary studies showed that the fall in HR did not have a significant effect on pulmonary or systemic hemodynamics, we selected to study only the asphyxic insult based on pressure change $(n=5)$. Five ventilated piglets without any insult served as controls to asphyxia. Because the hemodynamic data of the saline-instilled and ventilated control piglets without any insult did not differ from each other, the results are presented combined $(n=12)$.

The piglets were hemodynamically monitored and arterial blood samples for blood gas analysis were taken at baseline, 15 and $30 \mathrm{~min}$, and $1,2,3,4,5$, and $6 \mathrm{~h}$ after the insults. In addition, during asphyxia, repeated blood gas analyses were done. Blood glucose concentration was measured at baseline and every hour thereafter. To maintain the $\mathrm{PaO}_{2}$ between 10 and $14 \mathrm{kPa}$ and the $\mathrm{PaCO}_{2}$ between 4 and $6 \mathrm{kPa}$, supplemental oxygen was given and the frequency of the ventilator was adjusted if necessary. All piglets received initially $10 \mathrm{~mL} / \mathrm{kg}$ of isotonic sodium chloride solution (Ringer acetate) during the stabilization period. During the rest of the study, the piglets

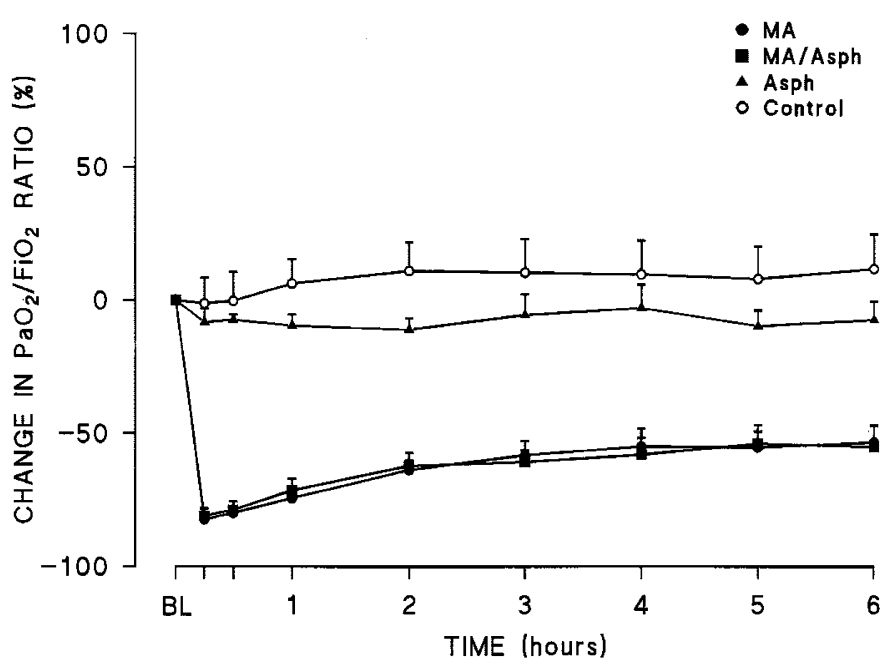

Figure 1. Percentile changes in the $\mathrm{PaO}_{2} / \mathrm{FiO}_{2}$ ratio from baseline $(B L)$ in newborn piglets during $6 \mathrm{~h}$ after meconium instillation $(M A, n=11)$, meconium instillation followed by asphyxia (MA/asph, $n=10)$, asphyxia alone $(A s p h, n=5)$, and controls $(n=12)$. Mean (SEM). MA and MA/asph groups are significantly different $(p<0.05)$ (repeated measures ANOVA with the mixed procedure) from asphyxia and control groups during the whole study period. 
were continuously infused with a $5 \%$ glucose solution at a rate of $12 \mathrm{~mL} / \mathrm{h}$. At the end of the experiment, the piglets were killed with an overdose of potassium chloride.

Statistical analysis. Statistical analysis was performed with the SAS statistical software (version 8.01; SAS Institute, Cary, NC, U.S.A.). The differences between the groups over time and separately at each time point were analyzed with repeated measures ANOVA using the mixed procedure. A $p$ value $<$ 0.05 was considered statistically significant. The results are expressed as mean (SD).

\section{RESULTS}

The average duration of asphyxia in the piglets with concurrent meconium administration was $5 \mathrm{~min}, 41 \mathrm{~s}(2 \mathrm{~min}, 33 \mathrm{~s}$, $\mathrm{SD})$ and in the group with isolated asphyxia 6 min, $7 \mathrm{~s}(58 \mathrm{~s})$. During the asphyxic insult, MABP dropped in the former group an average $45 \%(7 \%)$ and in the latter group $44 \%(3 \%)$. The arterial $\mathrm{pH}, \mathrm{PaO}_{2}$, and $\mathrm{PaCO}_{2}$ were similar at baseline in the asphyxia groups with and without preceding meconium instillation, and during the acute asphyxia dropped similarly to a mean $\mathrm{pH}$ of $6.90(0.09)$ and $6.80(0.07)$, a mean $\mathrm{PaO}_{2}$ of $1.4 \mathrm{kPa}$ $(0.3)$ and $1.7 \mathrm{kPa}(0.7)$, and a mean simultaneous rise of $\mathrm{PaCO}_{2}$ to $16.6 \mathrm{kPa}(3.6)$ and $16.2 \mathrm{kPa}(1.8)$, respectively. In the follow-up, no differences in the $\mathrm{pH}$ values between the groups were found. Meconium administration with and without acute asphyxia resulted in an acute and sustained decline in arterial oxygenation, indicated by a decreased ratio of $\mathrm{PaO}_{2} / \mathrm{FiO}_{2}$ (Fig. 1). In contrast, asphyxia alone did not alter the $\mathrm{PaO}_{2} / \mathrm{FiO}_{2}$ level (Fig. 1). Likewise, during the first hours after meconium instillation with and without asphyxia, arterial $\mathrm{PaCO}_{2}$ was higher than in the controls and asphyxic group, whereas asphyxia alone did not affect these values (data not shown).

Table 1. Hemodynamic data in newborn piglets at baseline (BL) and during 6 h after meconium instillation (MA, $n=11)$, meconium instillation followed by asphyxia (MA/asph, $n=10)$, asphyxia alone (asph, $n=5)$, and controls $(n=12)$.

\begin{tabular}{|c|c|c|c|c|c|c|c|c|c|}
\hline & $\mathrm{BL}$ & $15 \min$ & $30 \mathrm{~min}$ & $1 \mathrm{~h}$ & $2 \mathrm{~h}$ & $3 \mathrm{~h}$ & $4 \mathrm{~h}$ & $5 \mathrm{~h}$ & $6 \mathrm{~h}$ \\
\hline \multicolumn{10}{|l|}{ HR (beats/min) } \\
\hline MA & $217(32)$ & $199(32) \S \ddagger$ & $203(33)$ & $210(31)$ & $217(32)$ & $237(23)^{*}$ & $236(22)$ & $240(14) \S *$ & $239(9)^{*}$ \\
\hline $\mathrm{MA}+$ asph & $207(38)$ & $239(25)^{*}$ & $220(32)$ & $222(36)$ & $228(31)$ & $229(31)$ & $222(34)$ & $209(41)$ & $214(43)$ \\
\hline Asph & $233(13)$ & $246(8)^{*}$ & $210(35)$ & 237 (17) & $249(3)^{*}$ & $247(4)^{*}$ & 237 (14) & $229(21)$ & $220(24)$ \\
\hline Control & $203(23)$ & $212(24)$ & $212(29)$ & $214(37)$ & $212(41)$ & $209(37)$ & $212(32)$ & $206(27)$ & $193(34)$ \\
\hline \multicolumn{10}{|l|}{ CVP (mm Hg) } \\
\hline MA & $3(1)$ & $3(1)$ & $4(2)$ & $4(2)$ & $3(1)$ & $3(1)$ & $3(1)$ & $3(1)$ & $3(1)$ \\
\hline $\mathrm{MA}+$ asph & $3(1)$ & $3(1)$ & $3(1)$ & $3(1)$ & $3(1)$ & $3(1)$ & $3(1)$ & $3(1)$ & $3(1)$ \\
\hline Asph & $7(0)$ & $6(1)$ & $6(1)$ & $7(1)$ & $7(1)$ & $7(1)$ & $7(1)$ & $7(0)$ & $6(1)$ \\
\hline Control & $6(1)$ & $6(1)$ & $6(1)$ & $6(1)$ & $6(1)$ & $6(1)$ & $6(1)$ & $6(1)$ & $5(1)$ \\
\hline \multicolumn{10}{|l|}{ MPAP (mm Hg) } \\
\hline MA & $14(1)^{*} \div$ & $15(4) \S * \div$ & $16(3) \S \div$ & $17(2) \ddagger$ & $20(2)^{*}$ & $23(2)^{*}$ & $23(2)^{*}$ & $24(3)^{*}$ & $24(3)^{*}$ \\
\hline MA + asph & $15(3) \div$ & $20(4) \div$ & $19(4)$ & $19(3)$ & $22(6) *$ & $23(5)^{*}$ & $22(3) *$ & $22(3)$ & $23(4) *$ \\
\hline Asph & $21(4)^{*}$ & $24(4)^{*}$ & $20(3)$ & $21(3)$ & $20(3)^{*}$ & $21(3)^{*}$ & $22(4) *$ & $23(3)^{*}$ & $23(3)^{*}$ \\
\hline Control & $17(2)$ & $18(2)$ & $18(2)$ & $18(3)$ & $17(2)$ & $17(2)$ & $18(2)$ & $20(2)$ & $19(2)$ \\
\hline \multicolumn{10}{|l|}{ PWP (mm Hg) } \\
\hline MA & $3(1) * \div$ & $3(1)^{*} \div$ & $4(1)^{*} \div$ & $4(1)^{*}+$ & $5(1)^{*}+$ & $6(2) \S \div$ & $5(1)^{* \div}$ & $5(1)^{*} \div$ & $5(1)^{*} \div$ \\
\hline $\mathrm{MA}+$ Asph & $4(2) * \div$ & $4(2)^{*} \div$ & $4(1)^{*} \div$ & $4(1)^{*} \div$ & $4(1)^{*} \div$ & $4(1)^{* \div} \div$ & $4(1)^{*} \div$ & $5(1)^{*} \div$ & $5(1)^{*} \div$ \\
\hline Asph & $8(1)$ & $8(0)^{*}$ & $8(0)$ & $8(0)$ & $8(0)^{*}$ & $7(1)$ & $7(0)$ & $7(1)$ & $7(1)$ \\
\hline Control & $7(1)$ & $7(1)$ & $7(1)$ & $7(1)$ & $7(1)$ & $7(1)$ & $7(1)$ & $7(1)$ & $7(1)$ \\
\hline \multicolumn{10}{|l|}{ MABP (mm Hg) } \\
\hline MA & $71(11)$ & $77(15)$ & 77 (14) & 77 (15) & $80(11)$ & $79(9)$ & $70(9)$ & $64(7)$ & $59(12)$ \\
\hline $\mathrm{MA}+\mathrm{asph}$ & $76(16)$ & $85(16) *$ & $78(16)$ & $78(13)$ & $79(9)$ & $72(12)$ & $62(20)$ & $58(20)$ & $62(21)$ \\
\hline Asph & $68(11)$ & $81(13)$ & $69(5)$ & $77(6)$ & $83(10)$ & $75(9)$ & $63(3)$ & $59(8)$ & $53(12)$ \\
\hline Control & $68(13)$ & $73(11)$ & $75(14)$ & $77(13)$ & $77(15)$ & $73(14)$ & $71(13)$ & $68(14)$ & $63(17)$ \\
\hline \multicolumn{10}{|l|}{$\mathrm{CO}(\mathrm{L} / \mathrm{min})$} \\
\hline MA & $1.13(0.20)^{*}$ & $1.25(0.19)^{*}+$ & $1.23(0.18)^{*}+$ & $1.02(0.22) \div$ & $0.93(0.25)$ & $0.93(0.15)$ & $0.85(0.12)$ & $0.84(0.15)$ & $0.80(0.19)$ \\
\hline $\mathrm{MA}+$ asph & $1.27(0.23)^{*}$ & $1.38(0.34)^{*}+$ & $1.13(0.30) \div$ & $1.04(0.27) \dagger$ & $0.93(0.20)$ & $0.94(0.21)$ & $0.86(0.26)$ & $0.81(0.24)$ & $0.82(0.23)$ \\
\hline Asph & $1.09(0.16)$ & $0.90(0.10)$ & $0.74(0.06)^{*}$ & $0.77(0.14)$ & $0.77(0.17)$ & $0.75(0.12)$ & $0.76(0.16)$ & $0.75(0.21)$ & $0.64(0.90)$ \\
\hline Control & $1.04(0.24)$ & $1.02(0.19)$ & $0.99(0.19)$ & $0.93(0.25)$ & $0.86(0.24)$ & $0.90(0.30)$ & $0.80(0.16)$ & $0.76(0.16)$ & $0.70(0.20)$ \\
\hline \multicolumn{10}{|c|}{ PVR (mm Hg/L/min) } \\
\hline MA & $8.7(1.8)$ & $9.7(2.5) \ddagger$ & $9.8(3.2) \ddagger$ & $12.8(3.1)$ & $17.9(5.3)^{*}$ & $18.6(3.9)^{*}$ & $21.9(4.3)^{*}$ & $23.0(5.6)^{*}$ & $24.0(8.5)^{*}$ \\
\hline $\mathrm{MA}+\mathrm{asph}$ & $9.3(3.1)$ & $11.9(4.4) \ddagger$ & $13.8(5.2)$ & $15.4(6.2)$ & $20.3(8.1)^{*}$ & $21.5(7.6)^{*}$ & $22.6(7.3)^{*}$ & $23.0(6.7)^{*}$ & $23.7(5.0)^{*}$ \\
\hline asph & $12.6(4.2)$ & $18.2(5.8)^{*}$ & $15.8(4.1)$ & $17.8(7.0)$ & $17.0(6.8)$ & $19.3(7.0)^{*}$ & $20.7(10.0)^{*}$ & $23.5(10.1)$ & $26.4(10.2)^{*}$ \\
\hline control & $10.4(3.5)$ & $11.3(3.2)$ & $11.3(3.5)$ & $13.4(6.1)$ & $12.8(3.5)$ & $13.2(3.4)$ & $14.4(2.4)$ & $18.0(4.6)$ & $18.2(4.0)$ \\
\hline \multicolumn{10}{|c|}{$\mathrm{SVR}$ (mm Hg/L/min) } \\
\hline MA & $56.0(10.4)$ & $59.4(10.0) \ddagger$ & $61.1(15.0) \div$ & $73.6(17.6)$ & $88.6(27.6)$ & $84.5(19.5)$ & $80.7(14.2)$ & $74.2(12.1)$ & $71.2(13.6)$ \\
\hline $\mathrm{MA}+$ asph & $58.3(13.2)$ & $61.3(12.3) \div$ & $68.7(17.4)$ & $76.0(20.3)$ & $85.2(19.4)$ & 76.1 (16.9) & $70.2(17.4)$ & $68.9(18.1)$ & $73.1(18.3)$ \\
\hline Asph & $57.0(11.4)$ & $83.7(18.1)$ & $83.8(6.5)$ & $93.3(15.9)$ & $100.5(11.7)$ & $91.0(4.6)$ & $76.0(12.0)$ & $72.6(11.3)$ & $74.0(15.0)$ \\
\hline Control & $62.1(20.0)$ & $68.0(20.5)$ & $72.1(23.7)$ & $82.1(33.1)$ & $89.1(38.3)$ & $80.9(30.1)$ & $82.9(21.0)$ & $83.5(21.4)$ & $84.5(24.7)$ \\
\hline
\end{tabular}

Values are mean $(\mathrm{SD})$.

$* p<0.05$ vs controls.

$\S p<0.05 v s$ meconium instillation followed by asphyxia.

$\ddagger p<0.05$ vs asphyxia alone. 
Blood glucose concentrations were similar in all groups throughout the experiment, except a momentary increase $(+27 \%$ at $1 \mathrm{~h})$ after the asphyxic meconium insult (data not shown).

The absolute hemodynamic data in the different groups are presented in Table 1. Similar to previous findings from our laboratory (2), there was a progressive rise in MPAP after meconium instillation, being maximal $(80 \%$ from the baseline value) at $5 \mathrm{~h}$ (Fig. 2). Whereas asphyxia alone did not change MPAP, its combination to meconium instillation resulted in a progressive rise in MPAP, but the increase reached a significantly lower mean level than after isolated meconium insufflation $(48 \%$ versus $73 \%$ at $4 \mathrm{~h}$ and $50 \%$ versus $80 \%$ at $5 \mathrm{~h}$, respectively) (Fig. 2). Although asphyxia, similar to controls, increased the PVR to some extent during the experiment, there was a similarly exaggerated increase in PVR after meconium instillation with and without additional asphyxia (Fig. 2).

Whereas MABP after meconium instillation did not differ from the control values, combination of the meconium insult with asphyxia led, similar to isolated asphyxia, to a fall in MABP already at $4 \mathrm{~h}$ (Fig. 3). Although SVR after asphyxia alone was initially higher, and CO simultaneously lower (data not shown), when compared with meconium administration
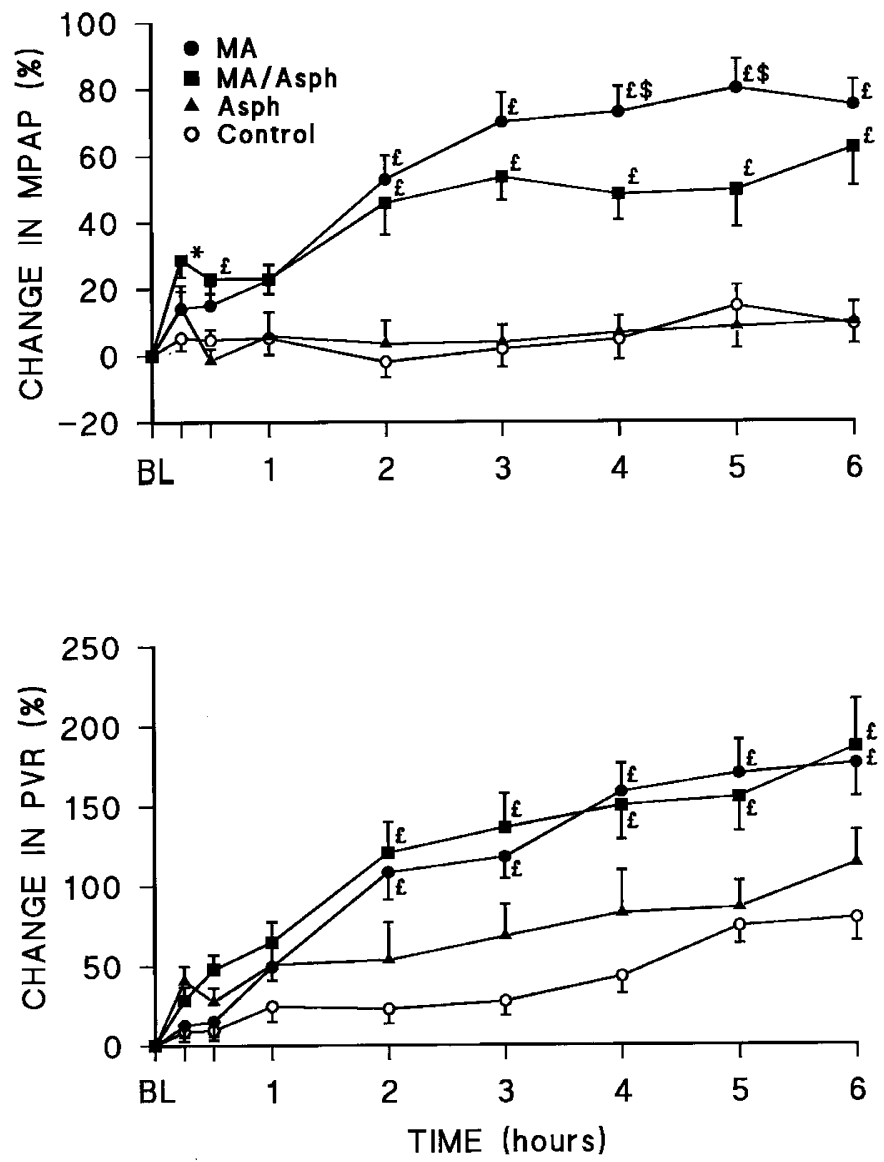

Figure 2. Percentile changes in MPAP and PVR from baseline $(B L)$ in newborn piglets during $6 \mathrm{~h}$ after meconium instillation $(M A, n=11)$, meconium instillation followed by asphyxia (MA/asph, $n=10)$, asphyxia alone (Asph, $n=5)$, and controls $(n=12)$. Mean (SEM). ${ }^{*} p<0.05$ vs controls; $£ p<0.05$ vs controls and asphyxia alone, ${ }^{\$} p<0.05 v s$ meconium instillation combined with asphyxia.
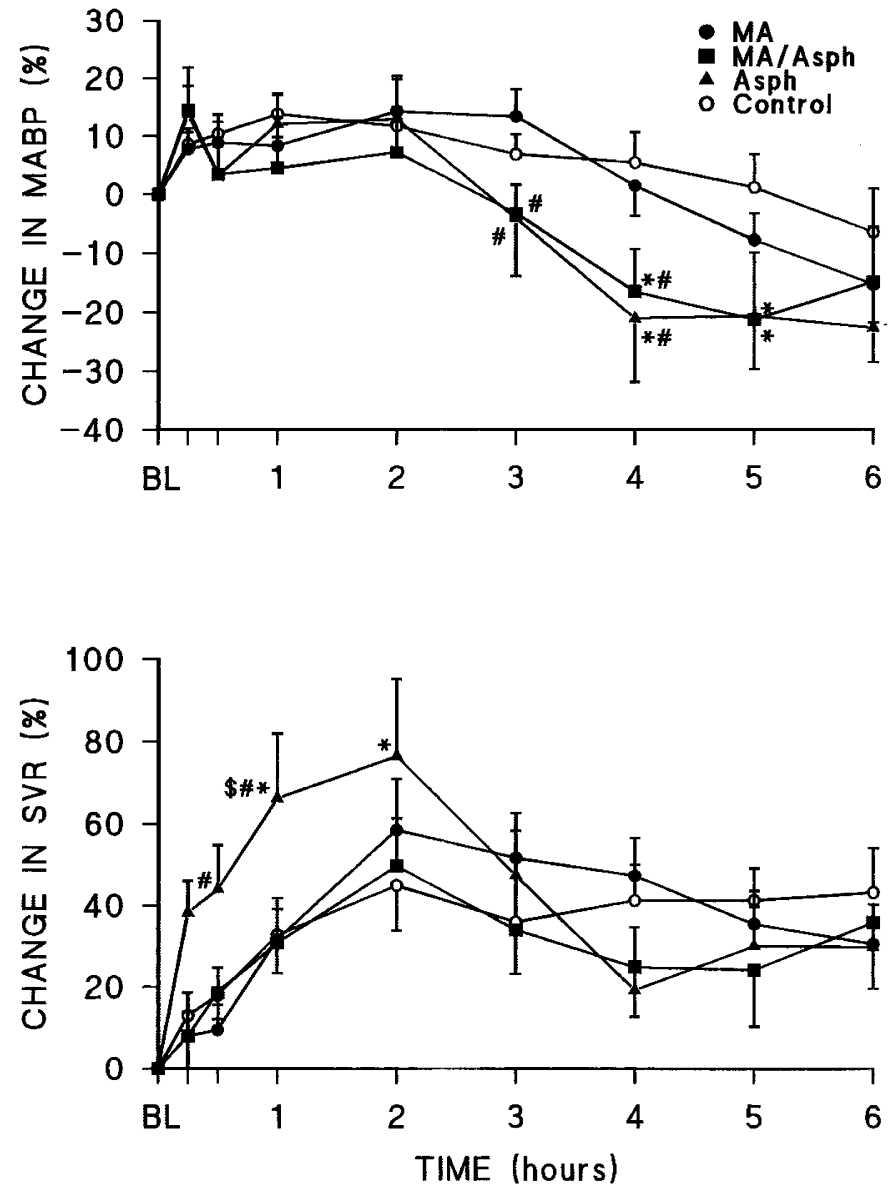

Figure 3. Percentile changes in MABP and SVR from baseline $(B L)$ in newborn piglets during $6 \mathrm{~h}$ after meconium instillation $(M A, n=11)$, meconium instillation followed by asphyxia (MA/asph, $n=10)$, asphyxia alone (Asph, $n=5)$, and controls $(n=12)$. Mean (SEM). ${ }^{*} p<0.05 v s$ controls, ${ }^{\$} p<0.05$ vs meconium instillation combined with asphyxia, ${ }^{*} p<$ $0.05 v s$ meconium instillation.

with and without asphyxia, there were no further differences in CO or SVR between the study groups (Fig. 3). Consequently, the ratio of PVR to SVR was elevated from $2 \mathrm{~h}$ onward after meconium insult with and without asphyxia (Fig. 4). An initial rise in HR $(18 \%$ at $1 \mathrm{~h})$ was further found after asphyxia combined to meconium insult, but no other differences were found in HR between the groups (data not shown). CVP was similar in all study groups (data not shown).

\section{DISCUSSION}

The present data indicate that acute asphyxia, when connected to meconium-induced parenchymal disease in newborn piglet lungs, does not potentiate the pulmonary hypertensive response or the level of arterial hypoxemia, but rather diminishes the pulmonary pressure level in the meconiumcontaminated lungs. At the same time, however, asphyxia seems to be mainly responsible for the accelerated fall in systemic arterial pressure after a combined insult. Although we describe here only the early phase after the insults, intrapulmonary thick meconium, used in this study, is shown to induce significant pulmonary circulatory and inflammatory reactions 


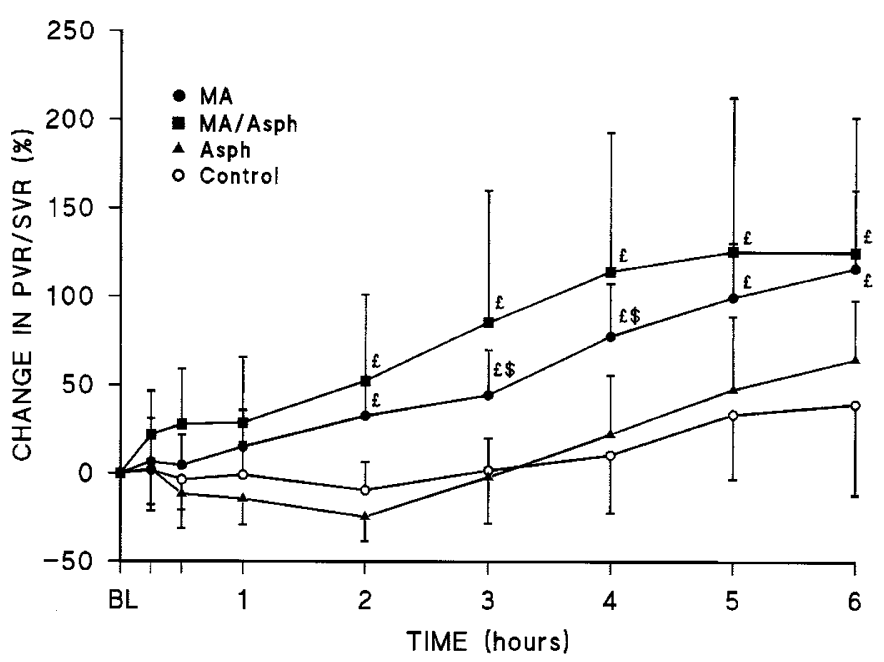

Figure 4. Percentile changes in the $\mathrm{PVR} / \mathrm{SVR}$ ratio from baseline $(B L)$ in newborn piglets during $6 \mathrm{~h}$ after meconium instillation $(M A, n=11)$, meconium instillation followed by asphyxia (MA/asph, $n=10)$, asphyxia alone (Asph, $n=5$,) and controls $(n=12)$. Mean (SD). $£ p<0.05 v s$ controls and asphyxia alone; ${ }^{\$} p<0.05$ vs meconium instillation combined with asphyxia.

already within that time period $(2,4)$. Furthermore, it has been reported earlier that the respiratory failure in asphyxic term infants with or without MAS reaches a peak during the first $8 \mathrm{~h}$ of life (10). Consequently, because piglet pulmonary vascular reactivity and morphology is similar to that in the human (17), the present results may be expected to adequately reflect hemodynamic changes in newborn infants with acute meconium aspiration and concomitant asphyxia. Nevertheless, because ductus arteriosus is shown to be functionally closed in the piglets at the age of $10 \mathrm{~d}(2)$, these observations are valid only for situation with no extrapulmonary ductal shunting.

The early pulmonary hemodynamic adaptation in asphyxic newborns with a progressing parenchymal lung disease, the main focus of our present study, is reported to be severely affected (10), but the interplay of these acute insults in the development of the circulatory perturbations has remained controversial $(1,11)$. In clinical surveys, the frequently evolving pulmonary hypertension in infants with severe MAS is commonly, but not always (8), related to the occurrence of fetal distress and pulmonary vascular structural and functional changes $(3,12,18)$. On the other hand, corroborating the present results, experimental works have shown that meconium instillation into the newborn lungs is able to produce significant pulmonary hypertensive reaction and severe oxygenation disturbances already within a few hours after the insult $(2,7)$. Whereas, as also evident from our data, acute asphyxia in newborns alone is not connected to a significant or sustained pulmonary hypertensive response, its combination with a parenchymal lung disease like MAS has been proposed to result in intense pulmonary hypertension in newborn infants (10). Our experimental data, however, indicate that this early rise in pulmonary vasomotor tone in meconium-contaminated neonatal lungs is not potentiated but rather moderated by additional asphyxia. A similar pressure-moderating trend was also described in meconium-contaminated lungs of newborn baboons that further showed, in contrast to our piglets, some improvement in oxygenation after concurrent asphyxia (19). The mechanisms of this modifying effect of concomitant asphyxia on pulmonary vascular tone remain uncertain, but alterations in pulmonary vasoreactivity, rather than the effect of hypoxemia or acidosis per se, may explain this finding. Because even profound perinatal asphyxia may not affect the ability of the newborn lungs to shunt blood from the atelectatic areas, as may be created by meconium obstruction (20), this modulation of the pulmonary vasomotor activity likely occurs in the nonobstructed areas of the affected lungs.

Progressive increase in PVR without a parenchymal lung disease is shown to result in a significant fall in systemic arterial pressure in adults and newborns, apparently through decreased left ventricular output $(21,22)$. Our present and earlier findings however indicate that the vascular hypertensive response in meconium-contaminated newborn lungs is not connected with significant changes in cardiac output or systemic arterial pressure $(2,23)$. Nevertheless, an exaggerated fall in systemic vascular pressure was observed when severe asphyxia was associated with the pulmonary hypertensive meconium insult. Because the same hypotensive effect was also seen after acute asphyxia without a parenchymal hypertensive lung disease, the fall of systemic arterial pressure in our piglets was unlikely caused by markedly compromised cardiac function resulting from reduced pulmonary perfusion. Our data are in contrast to an earlier report in newborn baboons suggesting no significant systemic hemodynamic effects after a combination of meconium and asphyxic insult, but different species and setup may have influenced the results (19). However, because we monitored only the right ventricular output, we cannot totally exclude the adverse effect of the profound asphyxic episode itself on the left ventricular function, proposed to be an important contributing factor to the neonatal respiratory failure (11). Consequently, asphyxia combined with a parenchymal lung disease may through hypoxemia and systemic hypotension further impair the organ function in the newborns, including cerebral vasoreactivity, and thereby place especially the brain at high risk for extension of injury $(24,25)$.

The intensity of the pulmonary artery hypertension and its attendant hypoxemia in the compromised newborn infants reflect well the severity of the respiratory failure and are, in their most severe forms, often refractory to various modes of treatment $(12,14,26)$. Diverse therapeutic approaches acting on the pulmonary and systemic vasomotor tone may additionally complicate the circulatory morbidity and may be deleterious to the injured tissues $(9,12,14,26)$. Consequently, excessive oxygen administration may through free radical production potentiate the effects of the initial insults, especially in the lung and brain (25). In line with earlier works (27), our circulatory data suggest that restricted oxygen use after acute asphyxia may be sufficient, whereas simultaneously initiated pulmonary hypertension may signal need for higher therapeutic oxygen concentrations. Although oxygen administration may not prevent the appearance of early systemic hypotension in a combined insult, hypoxemic pulmonary hypertension is supposed to protects the newborn organs against oxidative injury during resuscitative efforts after birth asphyxia (28). Thus, 
inasmuch as monitoring of the central hemodynamics may adequately reflect the newborn organ function and outcome, they should be taken into account in the management of compromised infants.

\section{CONCLUSION}

In conclusion, the present experimental data demonstrate that after a combined insult of meconium aspiration and acute asphyxia, the parenchymal lung disease is mainly responsible for the pulmonary dysfunction, in terms of changes in hemodynamics and oxygenation, whereas the acute asphyxic episode may potentiate the systemic hemodynamic perturbations. It is thus conceivable that the severe circulatory deteriorations induced by these two insults in combination may potentially have adverse effects on the function of multiple organ systems of the newborns and should be accordingly recognized in the therapeutic approaches of compromised infants.

\section{REFERENCES}

1. Wiswell TE, Bent RC 1993 Meconium staining and the meconium aspiration syndrome. Unresolved issues. Pediatr Clin North Am 40:955-981

2. Holopainen R, Soukka H, Halkola L, Kaapa P 1998 Meconium aspiration induces a concentration-dependent pulmonary hypertensive response in newborn piglets. Pediatr Pulmonol 25:107-113

3. Fox WW, Gewitz MH, Dinwiddie R, Drummond WH, Peckham GJ 1977 Pulmonary hypertension in the perinatal aspiration syndromes. Pediatrics 59:205-211

4. Davey AM, Becker JD, Davis JM 1993 Meconium aspiration syndrome: physiological and inflammatory changes in a newborn piglet model. Pediatr Pulmonol 16:101108

5. Cleary GM, Wiswell TE 1998 Meconium-stained amniotic fluid and the meconium aspiration syndrome. An update. Pediatr Clin North Am 45:511-529

6. Murphy JD, Vawter GF, Reid LM 1984 Pulmonary vascular disease in fatal meconium aspiration. J Pediatr 104:758-762

7. Trindade O, Goldberg RN, Bancalari E, Dickstein P, Ellison J, Gerhardt T 1985 Conventional vs high-frequency jet ventilation in a piglet model of meconium aspiration: comparison of pulmonary and hemodynamic effects. J Pediatr 107:115120

8. Perlman EJ, Moore GW, Hutchins GM 1989 The pulmonary vasculature in meconium aspiration. Hum Pathol 20:701-706
9. Kinsella JP, Abman SH 1995 Recent developments in the pathophysiology and treatment of persistent pulmonary hypertension of the newborn. J Pediatr 126:853864

10. Thibeault DW, Hall FK, Sheehan MB, Hall RT 1984 Postasphyxial lung disease in newborn infants with severe perinatal acidosis. Am J Obstet Gynecol 150:393-399

11. Perlman JM, Tack ED, Martin T, Shackelford G, Amon E 1989 Acute systemic organ injury in term infants after asphyxia. Am J Dis Child 143:617-620

12. Morin III FC, Stenmark KR 1995 Persistent pulmonary hypertension of the newborn. Am J Respir Crit Care Med 151:2010-2032

13. Carter BS, Haverkamp AD, Merenstein GB 1993 The definition of acute perinatal asphyxia. Clin Perinatol 20:287-304

14. Klesh KW, Murphy TF, Scher MS, Buchanan DE, Maxwell EP, Guthrie RD 1987 Cerebral infarction in persistent pulmonary hypertension of the newborn. Am J Dis Child 141:852-857

15. Scher MS, Klesh KW, Murphy TF, Guthrie RD 1986 Seizures and infarction in neonates with persistent pulmonary hypertension. Pediatr Neurol 2:332-339

16. Kusuda S, Shishida N, Miyagi N, Hirabayashi M, Kim TJ 1999 Cerebral blood flow during treatment for pulmonary hypertension. Arch Dis Child Fetal Neonatal Ed 80:F30-F33

17. Haworth SG, Hislop AA 1981 Adaptation of the pulmonary circulation to extrauterine life in the pig and its relevance to the human infant. Cardiovasc Res 15:108-119

18. Katz VL, Bowes Jr WA 1992 Meconium aspiration syndrome: reflections on a murky subject. Am J Obstet Gynecol 166:171-183

19. Cornish JD, Dreyer GL, Snyder GE, Kuehl TJ, Gerstmann DR, Null Jr DM, Coalson JJ, deLemos RA 1994 Failure of acute perinatal asphyxia or meconium aspiration to produce persistent pulmonary hypertension in a neonatal baboon model. Am J Obstet Gynecol 171:43-49

20. Berry D, Jobe A, Ikegami M, Seidner S, Pettenazzo A, Elkady T 1988 Pulmonary effects of acute prenatal asphyxia in ventilated premature lambs. J Appl Physiol 65:26-33

21. Belik J, Baron K, Light RB 1989 Central hemodynamic and regional blood flow changes in the newborn with right ventricular hypertension. Pediatr Res 26:548-553

22. Vlahakes GJ, Turley K, Hoffman JI 1981 The pathophysiology of failure in acute right ventricular hypertension: hemodynamic and biochemical correlations. Circulation 63:87-95

23. Korhonen KO, Seppanen MP, Kero PO, Kaapa PO 1999 Delayed adaptation of the pulmonary hemodynamics in infants with mild to moderate meconium aspiration syndrome. J Pediatr 134:355-357

24. Rosenberg AA 1988 Regulation of cerebral blood flow after asphyxia in neonatal lambs. Stroke 19:239-244

25. Piazza AJ 1999 Postasphyxial management of the newborn. Clin Perinatol 26:749 765 , ix

26. Sell EJ, Gaines JA, Gluckman C, Williams E 1985 Persistent fetal circulation Neurodevelopmental outcome. Am J Dis Child 139:25-28

27. Saugstad OD 2001 Resuscitation of the asphyxic newborn infant: new insight leads to new therapeutic possibilities. Biol Neonate 79:258-260

28. Ballot DE, Rothberg AD, Davies VA, Smith J, Kirsten G 1993 Does hypoxemia prevent brain damage in birth asphyxia? Med Hypotheses 41:344-347 